INRA Prod. Anim., 2000, 13 (3), 151-163

\section{S. INGRAND}

INRA SAD/ENA, Unité de Recherches sur les Herbi vores,

Thei $x, 63122$ St-Genès-Champanelle

email : ingrand@dermont.inra.fr

\section{Comportement alimentaire, quantités ingérées et performances des bovins conduits en groupe}

\section{Les éleveurs de bovins allaitants répartissent les vaches du troupeau dans différents lots en fonction de nombreux critères et aussi des contraintes structurelles : main d'œuvre, bâtiments, parcellaire. La composition des lots et le mode d'élevage ont des répercussions sur les performances des animaux, qu'on peut appréhender par leurs effets sur le comportement alimentaire et l'ingestion d'aliments.}

Les recommandations pour la constitution des lots de vaches formulées par les nutritionnistes sont basées sur des considérations liées au niveau de production zootechnique et au coût de la ration. II s'agit de regrouper en lots les animaux ayant les besoins nutrition-

\section{Résumé}

La hiérarchie sociale et les effets d'entraînement sont deux composantes importantes du comportement social qui influencent fortement le niveau d'ingestion et le comportement alimentaire des bovins conduits en groupe. Cet effet se traduit pour des animaux à besoins nutritionnels élevés ( animaux laitiers ou en croissance) par des niveaux d'ingestion supérieurs et une moindre efficacité alimentaire par rapport à des situations où les animaux sont alimentés individuellement (à l'attache ou en box individuel). Les caractéristiques des groupes ( compétition alimentaire, densité, nombre d'auges, effectif, stabilité dans le temps, caractéristiques des animaux) ont également des effets que nous avons cherché à quantifier. L'augmentation de la densité, la réduction de la largeur d'auge ou du temps d'accès à l'auge engendrent une diminution de la durée quotidienne d'ingestion et de la synchronisation de l'activité alimentaire, associée à une augmentation de la vitesse d'ingestion pouvant permettre le maintien du niveau d'ingestion, même lorsque le niveau de compétition est élevé. La taille du groupe a peu d'effet sur les composantes de l'ingestion des bovins et elle détermine surtout la vitesse à laquelle s'établit la hiérarchie sociale au sein du groupe. Les animaux aux besoins nutritionnels élevés ingèrent davantage, plus vite et plus longtemps que ceux aux besoins plus faibles, mais l'effet de l'hétérogénéité de ces besoins au sein d'un même groupe n'a pas été mesuré. Les études en fermes montrent pourtant que les lots gérés par les éleveurs de vaches allaitantes ne sont pas en majorité homogènes sur le plan des besoins nutritionnels, y compris durant la période des vêlages. Cela pose la question du risque d'altération des performances de ces animaux conduits en groupes hétérogènes. nels les plus similaires possibles, puis de définir, pour chacun de ces lots, une ration dont les apports alimentaires couvrent ces besoins au plus juste. La composition des lots est ensuite modifiée quand I'hétérogénéité intralot des besoins nutritionnels s'accroît.

En élevage bovin allaitant, l'association de contraintes et d'objectifs de production multiples conduisent les éleveurs à répartir les vaches dans les lots en fonction de nombreux critères qui ne correspondent pas, en majorité, à la recherche d'une augmentation de l'homogénéité intra-lot des besoins nutritionnels (Ingrand 1999). L'hétérogénéité intra-lot des besoins nutritionnels pose alors la question de l'évaluation des risques que prennent les éleveurs dans leurs choix de conduite quand le principe d'ajustement des apports alimentaires aux besoins des animaux n'est pas respecté. Ce risque est de deux ordres : risque d'accroissement du coût alimentaire en raison d'une surconsommation d'aliment par les animaux ayant les besoins les plus faibles et risque d'altération des performances zootechniques des animaux ayant les besoins les plus élevés. De telles approches ont déjà été effectuées pour les animaux laitiers pour préciser les conditions optimales de l'alimentation du troupeau (nombre, composition et niveau de rationnement des groupes) : voir par exemple Harb et al (1985) et Albright (1993) pour des 
vaches laitières, Bocquier et al (1995) pour des brebis laitières. Pour les bovins allaitants, il semble nécessaire de mieux connaître l'élaboration de la performance des animaux conduits en groupes hétérogènes sur le plan des besoins nutritionnels pour mesurer le risque d'altération de cette performance. II s'agit alors d'analyser la capacité des animaux à s'adapter selon les caractéristiques de leurs congénères et le type de conduite, principalement alimentaire, auquel le groupe est soumis.

Cet article se propose de faire le point sur ce que la notion de groupe signifie pour les nutritionnistes, notamment en matière de modifications possibles du comportement alimentaire et du niveau d'ingestion des bovins. L'objectif est de passer en revue les principaux facteurs de variation liés à la conduite en groupe en considérant l'ingestion comme une composante de la performance animale.

\section{1 / Conduite en groupe et comportement alimentaire des bovins}

\section{1 / Hiérarchie sociale au sein du groupe}

Quatre volets peuvent être distingués pour caractériser le comportement social des bovins (Albright et Arave 1997) : la communication, les phénomènes d'agressivité/soumission à l'égard des congénères ou de l'Homme, les phénomènes de leadership (effet d'entrầnement) et les relations de dominance entre les individus (hiérarchie sociale). Le phénomène de leadership se différencie de la notion de dominance qui caractérise la capacité d'un animal à inhiber le comportement d'autres individus dans le groupe (Syme et Syme 1975). La hiérarchie sociale formalise le comportement des individus appartenant au même groupe, permettant de diminuer la fréquence et l'intensité des interactions agonistiques : les affrontements directs sont remplacés par des attitudes de dominance/soumission (Dantzer et Mormède 1979).

Le rang hiérarchique des animaux dépend de plusieurs facteurs tels que l'âge (Kondo 1987), le poids vif, la présence de cornes, la taille, le sexe, l'ancienneté dans le groupe (Bouissou 1965). Les conditions d'hébergement et de conduite peuvent perturber l'expression de la hiérarchie sociale, mais ne la modifient pas (Wierenga 1990). Arave et al (1974) ont étudié la hiérarchie sociale dans deux groupes de 10 vaches, puis ont rassemblé d'un côté les 5 vaches dominantes et d'un autre côté les 5 vaches dominées de chaque groupe. La corrélation entre le rang social dans le nouveau groupe et le rang social dans le groupe d'origine a été de 0,86 . La hiérarchie sociale pour un groupe d'individus donné semble donc rester stable quand on introduit d'autres individus dans le groupe. D'autres études, impliquant des mâles, aboutissent à des conclusions plus nuancées. Par exemple, pour des taurillons laitiers au pâturage, Dalton et al (1967) n'ont pas réussi à définir clairement le classement hiérarchique des animaux dans des groupes de 25 têtes, en raison d'un individualisme très marqué des animaux, même au sein de groupes créés depuis 30 mois.

L'étude de Bouissou (1965) sur les mécanismes d'établissement de la hiérarchie sociale au sein de groupes de bovins a montré que l'établissement de cette hiérarchie est très rapide après la constitution du lot (dès la première heure), qu'elle s'établit souvent sans lutte entre les animaux, qu'elle est stable dans le temps et qu'elle est dans la plupart des cas simple et linéaire, bien qu'il puisse exister des formes plus complexes. La probabilité d'obtenir une hiérarchie linéaire est plus élevée pour des animaux adultes que pour des jeunes (Albright 1971) et elle décroît quand la taille du groupe augmente, mais reste forte pour des groupes de 7 ou 8 individus (Mestertongibbons et Dugatkin 1995). La stabilité du rang hiérarchique est également soulignée par Wierenga (1990) à l'échelle de plusieurs années; elle est plus grande dans les groupes de taille inférieure à 100 têtes, au sein desquels la reconnaissance de chaque individu est plus facile (Albright 1991).

Un essai portant sur des vaches laitières alimentées à l'auge a montré qu'à âge égal, les vaches dominantes ingèrent plus longtemps et ont des niveaux d'ingestion plus élevés que les vaches dominées, mais aucune liaison n'a été détectée entre le rang social et le niveau de production laitière (Krohn et Kongaard 1976, cités par Campling et Morgan 1981), ce qui a également été observé par Beilharz et al (1966). D'autres essais sur vaches laitières ont confirmé que, dans des situations de compétition pour l'accès à la nourriture, les animaux dominants ont des durées quotidiennes d'ingestion supérieures à celles des animaux dominés, mais leurs niveaux d'ingestion ne sont pas forcément plus élevés. La corrélation entre le niveau d'ingestion et le rang social s'accroît avec le niveau de compétition alimentaire (Friend et Polan 1974, Friend et al 1977, Leaver et Yarrow 1980, Harb et al 1985). Pour des taurillons et des bœufs nourris à l'aide d'un distributeur unique, les animaux dominants font moins de repas par jour que les dominés ( 15 vs $19, P<0,05)$ mais avec une durée d'ingestion plus élevée ( 87 vs $78 \mathrm{~min} / \mathrm{j}$, $\mathrm{P}<0,05$; Stricklin et Gonyou 1981). Le comportement respectif d'animaux dominants et dominés a également été analysé par Manson et Appleby (1990) pour un troupeau de 45 vaches laitières recevant une ration complète à volonté. La distance entre deux vaches au cornadis est déterminée par leurs rangs sociaux (figure 1). Le même type d'observations a été réalisé pour l'occupation de logettes par des vaches laitières : les vaches de rang social élevé utilisent les logettes les plus proches de la zone de distribution de l'aliment et voisines les unes des autres (Friend et Polan 1974). 
Figure 1. Effet du rang social sur la distance entre vaches: moyenne de la distance avec le voisin le plus proche pour différentes combinaisons hiérarchiques (d'après Manson et Appleby 1990).

$\mathrm{B}, \mathrm{M}$ et $\mathrm{H}=$ respectivement bas, moyen et haut rang social

Distance au voisin le plus proche (nombre de cornadis)

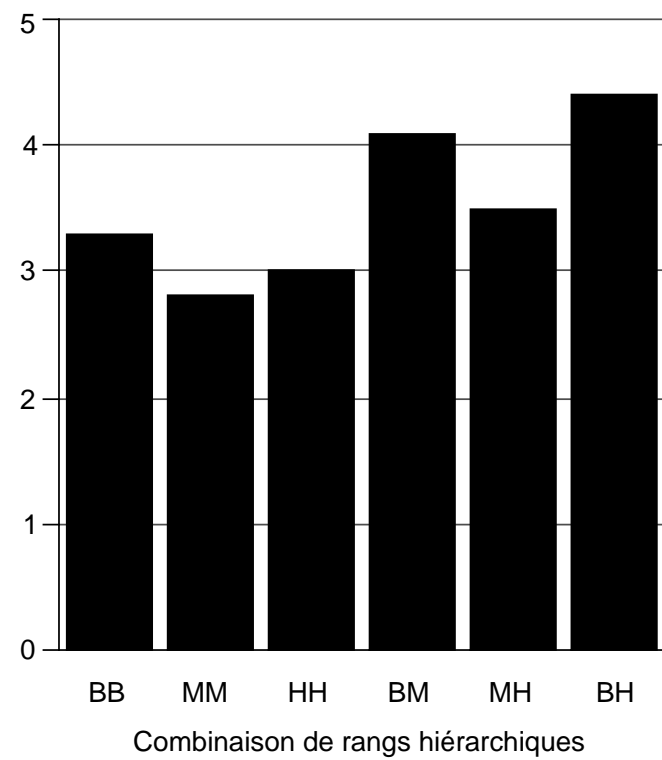

\section{2 / Synchronisation des activités au sein du groupe}

Chez les bovins, l'activité d'ingestion est essentiellement diurne, que ce soit au pâturage ou en bâtiments. Deux pics d'ingestion ont lieu au lever et au coucher du soleil. Le pic le plus important (en durée et en quantités ingérées) est celui du soir, entre $15 \mathrm{~h} 00$ et $18 \mathrm{~h} 00$ (Gonyou et Stricklin 1981, Rook et Huckle 1995). Les proportions moyennes de quatre activités (alimentation, abreuvement, station debout, station couchée) pour 324 taurillons répartis en 12 groupes de 27 têtes entre janvier et mai (Gonyou et Stricklin 1984), ont été respectivement de 9,8 - 1,9-27,4 et $60 \%$ Les profils quotidiens pour chacune de ces activités ont evolué en fonction des heures de lever et de coucher du soleil. Des vaches laitières en stabulation libre ne modifient pas leur rythme d'activité lorsqu'elles sont éclairées en continu par comparaison à $18 \mathrm{~h}$ d'éclairement par jour (Durst et al 1993). La distribution de la nourriture joue un rôle aussi important que la photopériode pour des animaux hébergés en bâtiments (Welch et Hooper 1988) et c'est elle qui déclenche le début des périodes d'ingestion correspondant aux grands repas. La composition du groupe peut egalement modifier l'activité alimentaire : Dolezal (1984, cité par Albright et Arave 1997) a constaté un décalage de l'activité d'ingestion vers la nuit après avoir introduit des individus étrangers dans des lots de taurillons à l'engrais.

L'effet des facteurs de synchronisation des activités (leadership, mobilité des animaux) observé au pâturage est très atténué en bâtiments (Metz et Wierenga 1987, Miller et
Figure 2. Effet de la réduction du nombre d'accès à l'aliment (portillons) sur le rythme et la synchronisation de l'ingestion au sein d'un troupeau de 19 vaches laitières alimentées avec de l'ensilage d'herbe distribué à volonté à $6 \mathrm{~h} 30,11 \mathrm{~h} 30$, $15 h 30$ et 21 h30 (d'après Metz et Wierenga 1987).

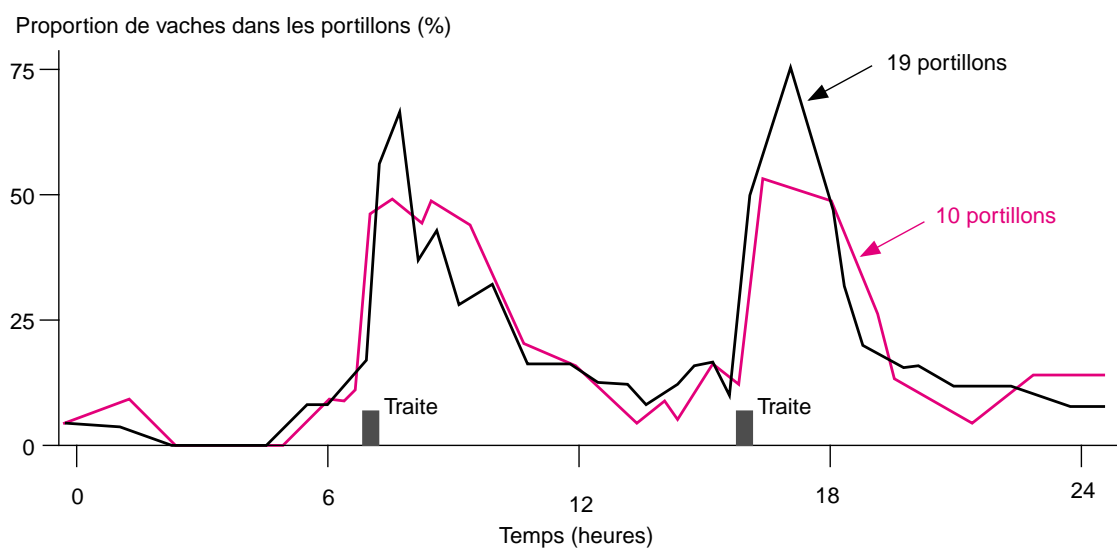

Wood-Gush 1991, Krohn et al 1992, Krohn 1994). En particulier à l'attache, le manque de place disponible induit une perturbation mutuelle quand les animaux voisins se lèvent ou se couchent. Dans l'essai de Miller et Wood-Gush (1991), les vaches laitières en stabulation libre ont été moins synchronisées qu'au pâturage, bien qu'elles aient disposé de portillons électroniques individuels et de logettes en nombre supérieur au nombre de vaches.

Pour ce qui concerne l'ingestion, la synchronisation semble être très stable chez les bovins, même en situation de forte compétition alimentaire (figure 2 ; Metz et Wierenga 1987). Selon ces auteurs, la synchronisation observée au sein des groupes implique que la compétition pour différentes activités (alimentation, repos) commence bien avant I'utilisation à $100 \%$ des structures (auges ou logettes de couchage). L'optimisation de l'utilisation des installations supposerait que chaque animal puisse avoir son propre rythme d'activité, différent et indépendant de celui de ses congénères.

\section{2 / Comparaison entre la conduite individuelle et la conduite en groupe}

L'effet des interactions sociales au sein $d u$ groupe sur le comportement alimentaire des bovins a été mis en évidence par des études comparant la conduite collective (stabulation libre) et la conduite individuelle (attache ou box individuel).

\section{1 / Niveau d'ingestion}

Dans la plupart des études, les quantités ingérées par les animaux sont supérieures quand ils sont conduits en groupe (figure 3 ). La différence observée par exemple par Coppock et al (1972) entre des vaches laitières alimentées à l'attache ou en stabulation libre a été de $7 \%$ en faveur de la stabulation libre, sans modification du niveau de production laitière, de composition du lait, ni de poids vif des animaux. Dans l'étude de

\section{Les quantités ingérées par les anima ux sont plus élevées lorsqu'ils sont alimentés en groupe.}


Tableau 1. Effet du mode de stabulation, entravée ou libre, sur les quantités ingérées (QI en kg de MS/j) par des génisses de race Pie-Noire, Tarine ou Salers, selon leur âge (d'après D'Hour et al 1991).

\begin{tabular}{|c|c|c|c|c|c|c|}
\hline Race & \multicolumn{2}{|c|}{ Pie-Noire } & \multicolumn{2}{|c|}{ Tarine } & \multicolumn{2}{|c|}{ Salers } \\
\hline Stabulation & entravée & libre & entravée & libre & entravée & libre \\
\hline $\begin{array}{l}\text { Génisses « } 1 \text { an » } \\
\text { Effectifs } \\
\text { QI par animal } \\
\text { QI/100 kg de poids vif }\end{array}$ & $\begin{array}{c}7 \\
5,91 \\
1,89\end{array}$ & $\begin{array}{c}7 \\
5,71 \\
1,82\end{array}$ & $\begin{array}{c}8 \\
4,96 \\
1,91\end{array}$ & $\begin{array}{c}8 \\
5,69 \\
2,06\end{array}$ & $\begin{array}{c}8 \\
5,16 \\
1,63\end{array}$ & $\begin{array}{c}7 \\
5,57 \\
1,77\end{array}$ \\
\hline $\begin{array}{l}\text { Génisses « } 2 \text { ans » } \\
\text { Effectifs } \\
\text { QI par animal } \\
\text { QI/100 kg de poids vif }\end{array}$ & $\begin{array}{c}7 \\
7,21 \\
1,54\end{array}$ & $\begin{array}{c}7 \\
7,65 \\
1,68\end{array}$ & $\begin{array}{c}8 \\
6,12 \\
1,53\end{array}$ & $\begin{array}{c}8 \\
7,43 \\
1,82\end{array}$ & $\begin{array}{l}- \\
- \\
-\end{array}$ & $\begin{array}{l}- \\
- \\
-\end{array}$ \\
\hline
\end{tabular}

Figure 3. Comparaison des quantités ingérées par des bovins à viande à l'engrais et des bovins laitiers (génisses et vaches), à l'attache et en stabulation libre (d'après Ingvartsen et Andersen 1993).

Consommation en stabulation libre (kg MS / j)

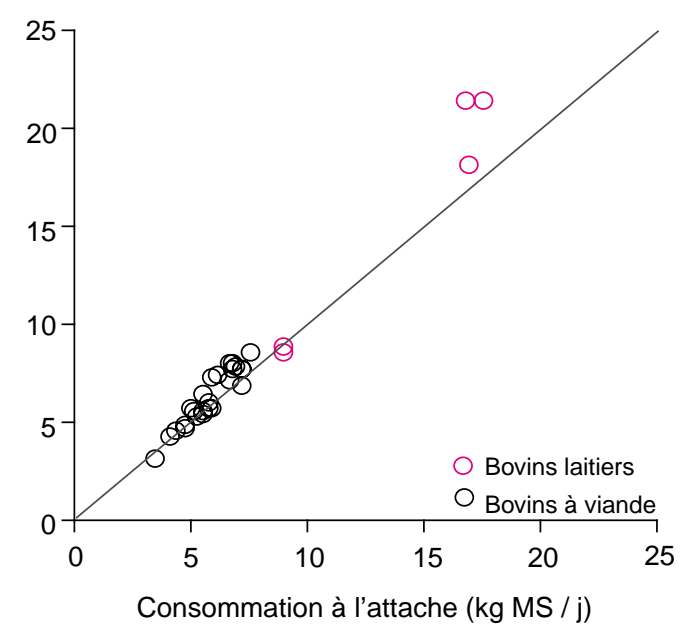

D'Hour et al (1991) sur des génisses laitières en croissance, le niveau d'ingestion s'est accru de $10 \%$ en moyenne en stabulation libre par rapport à la stabulation entravée (tableau 1). Phipps et al (1983) ont obtenu un accroissement de consommation de $9 \%$, associé à un gain de poids vif, chez des vaches laitières en stabulation libre qui sont passées d'une alimentation individuelle avec des portillons électroniques à une alimentation collective avec une auge commune.
Concernant les bovins de race à viande, les taurillons et les bœufs en croissance en groupe et en l'absence de compétition ingerent davantage que lorsqu'ils sont attachés, mais l'écart n'est pas tres important : $+4 \%$ en moyenne sur 13 comparaisons (revue d'Ingvartsen et Andersen 1993). L'étude de Broadbent et al (1970), sur des bœufs frisons soumis à quatre conduites alimentaires différentes, montre que le niveau d'ingestion et l'indice de consommation sont plus élevés avec une alimentation en groupe (tableau 2). Un essai conduit avec 47 vaches Charolaises taries ou en lactation a abouti aux résultats inverses pour les vaches en lactation, à savoir un niveau d'ingestion moindre en stabulation libre par rapport à l'attache $(-1,1 \mathrm{~kg} M S / \mathrm{j})$, aucune difference n'ayant été mesurée pour les vaches taries (Ingrand et al 1999).

Les niveaux d'ingestion supérieurs observés pour des animaux conduits en groupe sont expliqués par la stimulation réciproque (effet d'entraînement) et par des dépenses énergétiques qui seraient plus importantes, bien qu'une étude de Gustafson et al (1993) n'ait pas permis de mettre en évidence l'effet de l'exercice physique sur les quantités ingérées par des vaches laitières. Selon Albright (1993), plusieurs facteurs «psychologiques » entrent également en compte et le bien-être des animaux serait mieux assuré au sein d'un groupe. A l'inverse, les facteurs susceptibles d'entraîner une diminution des quantités ingérées en groupe sont liés à l'espace disponible (à la compétition en général) et à la motivation pour effectuer le déplacement vers les auges.

Tableau 2. Consommation d'aliment, gain de poids vif et efficacité alimentaire mesurés sur des bœufs à l'engrais selon différentes modalités de conduite (d'après Broadbent et al 1970).

\begin{tabular}{|c|c|c|c|}
\hline & $\begin{array}{l}\text { Consommation } \\
\text { d'aliment }(\mathrm{kg} / \mathrm{j})\end{array}$ & \begin{tabular}{|c|} 
Vitesse de \\
croissance $(\mathrm{kg} / \mathrm{j})$
\end{tabular} & $\begin{array}{c}\text { Efficacité } \\
\text { alimentaire }^{(1)}\end{array}$ \\
\hline $\begin{array}{l}\text { Hébergés et alimentés en groupe } \\
\text { moyenne } \\
\text { écart type } \\
\text { Hébergés en groupe et alimentés individuellement (collier) } \\
\text { moyenne } \\
\text { écart type } \\
\text { Hébergés en groupe et alimentés individuellement (harnais) } \\
\text { moyenne } \\
\text { écart type } \\
\text { Hébergés et alimentés individuellement } \\
\text { moyenne } \\
\text { écart type }\end{array}$ & $\begin{array}{c}5,62 \\
0,23 \\
\\
5,72 \\
0,33 \\
\\
5,81 \\
0,18\end{array}$ & $\begin{array}{c}1,48 \\
0,05 \\
\\
1,39 \\
0,06 \\
\\
1,46 \\
0,06\end{array}$ & $\begin{array}{c}4,19 \\
0,21 \\
\\
4,07 \\
0,17\end{array}$ \\
\hline
\end{tabular}

(1) $\mathrm{kg}$ d'aliment / kg de croît

INRA Productions Ani males, jui llet 2000 


\section{2 / Comportement alimentaire}

Les comparaisons entre la conduite individuelle et la conduite en groupe ont également porté sur certaines composantes de l'ingestion. Fuller (1928, cité par Albright 1993), avec des vaches nourries au foin à volonté, a obtenu des durées d'ingestion plus longues en box collectifs : $6 \mathrm{~h}$ contre seulement $3 \mathrm{~h}$ à I'attache, cette dernière valeur semblant d'ailleurs très faible. Les données de Munksgaard et Simonsen (1995), comparant des vaches laitières conduites en cases collectives par groupes de 4 animaux ou à l'attache ne montrent pas de différences de durée d'ingestion entre les deux traitements ( $5 \mathrm{~h} 19$ à l'attache et $4 \mathrm{~h} 45$ en cases collectives, soit tout de même 34 min d'écart). La fréquence d'ingestion a été plus élevée en case collective $(37,4$ séquences d'ingestion vs 27,1 à l'attache) et les durées et les fréquences de rumination ont été équivalentes entre les deux traitements. Harb et al (1985) ont obtenu avec des vaches laitières des vitesses d'ingestion de $43 \mathrm{~g} / \mathrm{min}$ en moyenne à l'attache, de $71 \mathrm{~g} / \mathrm{min}$ en stabulation libre avec 5 auges pour 10 vaches et de $57 \mathrm{~g} / \mathrm{min}$ en stabulation libre avec 10 auges pour 10 vaches. Les résultats semblent également dépendre du stade physiologique (Ingrand et al 1999) : des vaches Charolaises taries $(n=24)$ ont augmenté leur durée d'ingestion quotidienne en stabulation libre par rapport à l'attache (respectivement $5 \mathrm{~h} 20$ et $4 \mathrm{~h} 45$ ) à niveau d'ingestion équivalent, alors que leurs homologues en lactation ( $n=23)$ n'ont pas modifié leur durée d'ingestion ( 5 h50) pour un niveau d'ingestion moindre en stabulation libre.

Ainsi, la conduite en groupe semble entraîner une diminution de la durée quotidienne d'ingestion, une augmentation de la vitesse d'ingestion et une diminution du fractionnement de l'activité alimentaire.

\section{3 / Performances zootechniques}

Des performances de croissance plus faibles sont observées pour des veaux conduits en boxes par deux en comparaison avec des veaux conduits dans des cases individuelles (Hanekamp et al 1994). Dans la revue d'Ingvartsen et Andersen (1993), les niveaux d'ingestion supérieurs en groupe de bovins en croissance se traduisent par une moindre efficacité alimentaire : $+4 \%$ d'indice de consommation. Concernant la composition des carcasses, une étude de Nour et Thonney (1987) a montré que des bœufs (races Angus et Holstein) en cases individuelles couvertes $\left(5,28 \mathrm{~m}^{2}\right.$ par animal) ont eu une progression plus rapide de la teneur en muscles dans la carcasse que les animaux en cases collectives à l'extérieur $\left(23 \mathrm{~m}^{2}\right.$ par tête). Enfin, la conduite en groupe de génisses dans le jeune âge ne semble pas avoir d'effet sur la vitesse de croissance et sur le niveau de production laitière ultérieur (Arave et al 1992).

\section{3 / E ffets de la compétition alimentaire et de la densité sur le comportement alimentaire}

Pour un même type de ration, la durée et le profil d'ingestion dépendent du niveau de compétition alimentaire au sein du groupe. En situation de compétition alimentaire, l'accessibilité de la ration peut être un facteur plus important que la quantité d'aliment disponible. Indépendamment de l'accès à la nourriture, l'espace disponible pour chaque animal (densité) peut également constituer une contrainte qui va influencer les paramètres du comportement alimentaire, le niveau d'ingestion et finalement les performances zootechniques.

\section{1 / Temps d'accès à l'auge}

Les situations de compétition au sein d'un groupe peuvent être simulées avec des animaux conduits en cases individuelles en réduisant l'accès à la nourriture à quelques heures par jour : il s'agit alors de vérifier dans quelle mesure les animaux sont capables d'augmenter leur vitesse d'ingestion pour maintenir leur niveau d'ingestion quotidien. Plusieurs essais de ce type sont récapitulés à la figure 4. Le niveau d'ingestion diminue en moyenne de 15 à $20 \%$ (jusqu'à $33 \%$ avec une ration sans fourrage, Bines et Davey 1970) quand le temps d'accès à la nourriture est restreint à 4 ou $5 \mathrm{~h}$ par jour, alors qu'il est peu affecté quand le nombre d'auges disponibles diminue. L'augmentation de la vitesse d'ingestion ( +5 à $15 \mathrm{~g} / \mathrm{min}$ selon les essais) ne compense jamais la réduction du temps d'accès à l'auge.

\section{2 / Largeur d'auge}

Pour des animaux conduits en stabulation libre, la largeur d'auge minimale, en deçà de laquelle les quantités individuelles ingérées diminuent, dépend du temps d'accès à la nourriture. Ainsi, Leaver et Yarrow (1980) ont montré qu'avec un temps d'accès de $7 \mathrm{~h} / \mathrm{j}$, le passage de 40 à $20 \mathrm{~cm}$ de largeur d'auge par animal (génisse laitière de 17 mois) a entraîné une baisse de la durée d'ingestion de 180 à $130 \mathrm{~min} / \mathrm{j}$ et une réduction de $4 \%$ des quantités ingérées. Avec $50 \mathrm{~cm}$ par animal, la réduction du temps d'accès de 5 à $3 \mathrm{~h}$ par jour a entraîné une réduction de la durée d'ingestion de 156 à $124 \mathrm{~min} / \mathrm{j}$ et une réduction de $7 \%$ des quantités ingérées. Dans les deux cas, l'augmentation de la vitesse d'ingestion des animaux n'a que partiellement compensé l'augmentation du niveau de compétition alimentaire. Friend et al (1977) n'ont observé une diminution des durées et des niveaux d'ingestion qu'en decà de $20 \mathrm{~cm}$ d'auge par animal (vaches laitières). Au-delà de $20 \mathrm{~cm}$ par animal et jusqu'à $50 \mathrm{~cm}$, les deux paramètres n'ont pas varié. Lawrence (1994) donne des valeurs recommandées pour des largeurs d'auges qui varient de 40 à $70 \mathrm{~cm}$ selon le

\author{
Chez les animaux \\ en stabulation \\ libre, la vitesse \\ d'ingestion est plus \\ élevée et la durée \\ quotidienne \\ d'ingestion et le \\ nombre de repas \\ plus faibles que \\ chez les a nimaux \\ alimentés individu- \\ ellement.
}


Figure 4. Effet du nombre d'auges disponibles pour des vaches laitières et des taurillons (trois graphiques du haut) et du temps d'accès à l'auge pour des vaches laitières taries (trois graphiques du bas, adapté de Campling et Morgan 1981) sur les quantités ingérées, la durée et la vitesse d'ingestion.

X Ensilage d'herbe ; taurillons (Corkum et al 1994)

- Ensilage d'herbe ; vaches laitières (Harb et al 1985)

A Ensilage d'herbe ; vaches laitières (Harb et al 1985)

Quantités ingérées (kg MS / j)

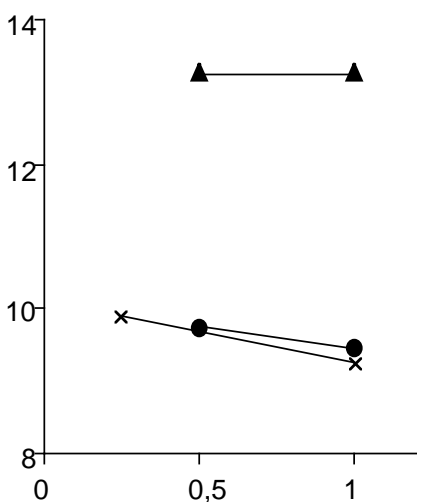

$\square$ Foin (Freer et al 1962)

$\Delta$ Foin (Suzuki et al 1970)

O Foin (Campling 1966)

Quantités ingérées ( $\mathrm{kg} \mathrm{MS} \mathrm{/} \mathrm{j)}$

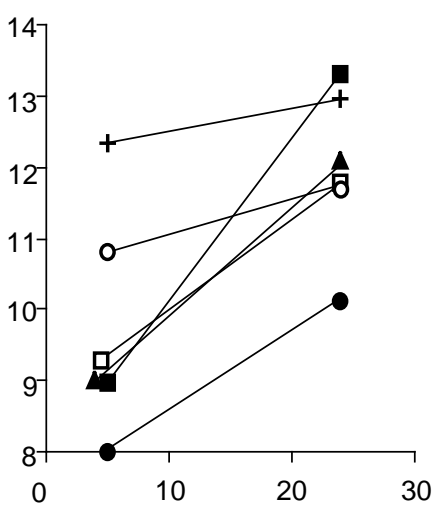

Durée d'ingestion ( $\min / \mathrm{j})$

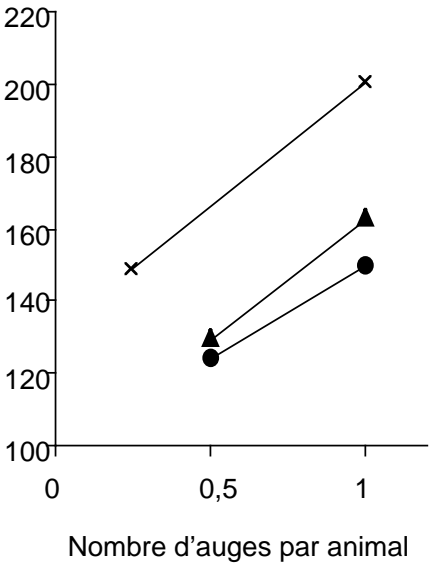

Nombre d'auges par animal
Vitesse d'ingestion (g MS / min)

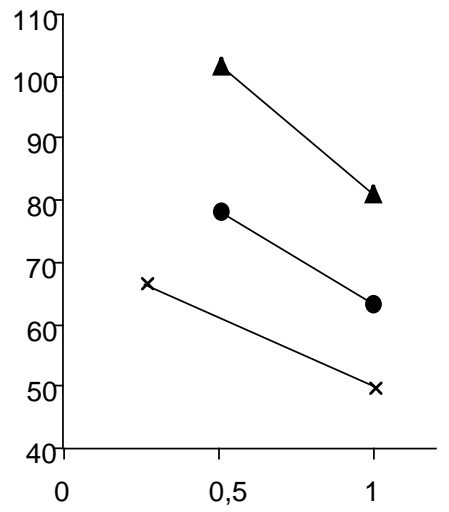

+ Ration complète $50 \%$ fourrage (Bines et Davey 1970)

Ration complète sans fourrage (Bines et Davey 1970)

Ensilage (Campling 1966)
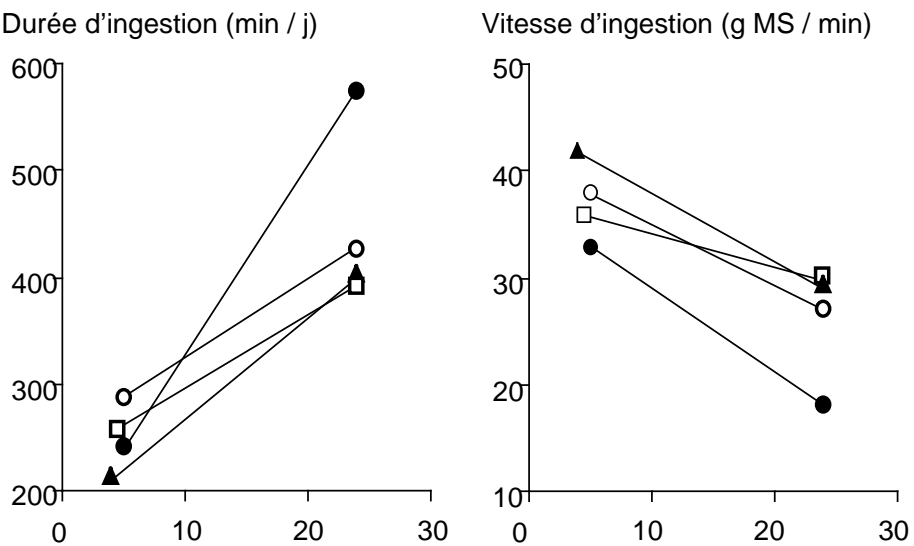

Temps d'accès à l'auge $(\mathrm{h} / \mathrm{j})$

Tableau 3. Largeurs d'auges recommandées pour des bovins de races à viande selon leur poids (d'après Lawrence 1994).

\begin{tabular}{|c|c|}
\hline Poids vif $(\mathrm{kg})$ & $\begin{array}{c}\text { Largeur d'auge } \\
\text { (mm/animal) }\end{array}$ \\
\hline 200 & 400 \\
300 & 500 \\
400 & 550 \\
500 & 600 \\
600 & 650 \\
700 & 700 \\
800 & 750 \\
\hline
\end{tabular}

poids vif des animaux (tableau 3). Ces valeurs sont élevées par rapport à celles testées dans les essais précédemment cités, mais l'auteur précise qu'elles peuvent être réduites de $75 \%$ dans le cas d'une alimentation disponible à volonté.

\section{3 / Nombre d'auges}

La compétition alimentaire peut également être induite par le nombre de distributeurs disponibles. Ainsi, Corkum et al (1994) ont testé trois niveaux de compétition différents pour des groupes de 12 bœufs à l'engrais conduits en stabulation libre en faisant varier le nombre de portillons électroniques par groupe. Sans compétition, les animaux ont effectué les repas les plus longs et les séquences d'ingestion les moins nombreuses.

INRA Producti ons Ani males, jui llet 2000 
Figure 5. Profil d'ingestion pour des lots de taurillons et de bœufs en stabulation libre (15 têtes/lot) alimentés à l'auge ou avec un seul distributeur d'aliment pour le lot (d'après Gonyou et Stricklin 1981).

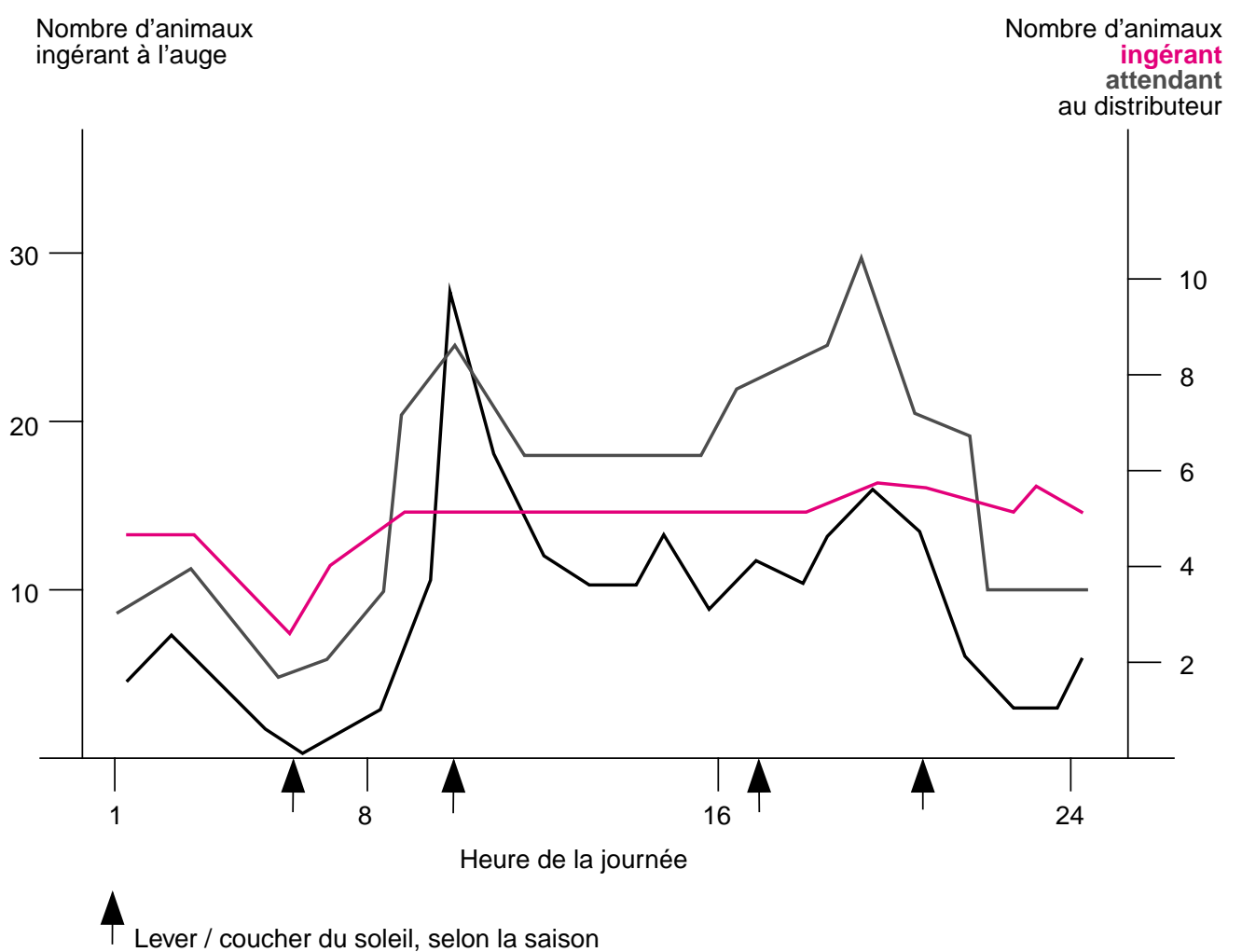

La compétition la plus forte a engendré les durées d'ingestion quotidienne les plus faibles mais avec un niveau d'ingestion equivalent au cas précédent. La plus faible durée d'ingestion a donc été compensée par une vitesse d'ingestion plus élevée (figure 4) et les vitesses de croissance ont été similaires. Les animaux dominés n'ont pas été désavantagés en situation de forte compétition. Pour le même type d'essai réalisé sur des vaches laitières (Harb et al 1985), les temps moyens d'ingestion ont été de $204 \mathrm{~min} / \mathrm{j}$ avec des auges individuelles vs $124 \mathrm{~min} / \mathrm{j}$ seulement avec 5 auges pour 10 vaches et $150 \mathrm{~min} / \mathrm{j}$ avec une auge par vache en accès libre, sans modification du nombre de séquences d'ingestion. Dans un autre essai des mêmes auteurs, la réduction du nombre d'auges a seulement entraîné la diminution de la durée d'ingestion. La vitesse d'ingestion moyenne dans les deux essais a fortement augmenté avec le niveau de compétition : $+65 \%$ et $+27 \%$ respectivement dans I'essai 1 et 2 . Cette augmentation a surtout été spectaculaire pour les vaches dominées, certaines ayant doublé leur vitesse d'ingestion d'un traitement à l'autre. De la même manière, Gonyou et Stricklin (1981) ont comparé le comportement alimentaire de groupes de 15 têtes (taurillons et bœufs) alimentés soit à l'auge (collectivement), soit par l'intermédiaire d'un poste unique de distribution. Les résultats n'ont pas montré de différences de quantités ingérées ni de performances de croissance, après une période d'adaptation. Cependant, les profils d'ingestion à l'échelle de la journée sont différents (figure 5) : deux pics d'ingestion sont observés pour les animaux alimentés à l'auge, au moment de la distribution de la ration, alors que l'utilisation du distributeur unique est pratiquement constante sur 24 heures. Chez les animaux nourris à l'auge, la vitesse d'ingestion est plus élevée.

\section{4 / Espace disponible pour les animaux}

La trop forte densité au sein d'un groupe de bovins entraîne des réactions d'agression plus fréquentes en raison de la forte probabilité de rencontres et du faible espace pour que l'animal agressé puisse s'échapper (Metz et Wierenga 1987). L'effet négatif d'une forte densité a également été montré chez des ovins et des cerfs au pâturage (Blanc et al 1999). L'espace disponible nécessaire aux animaux dépend de la zone considérée : il doit être plus important autour des lieux d'alimentation et d'abreuvement où la compétition est plus forte qu'ailleurs. Le temps passé dans ces zones est généralement plus important pour les animaux dominants que pour les animaux dominés (Metz et Wierenga 1987).

Metz et Mekking (1984) ont montré qu'en situation de faible compétition pour l'accès à la nourriture, la densite n'affecte pas le comportement alimentaire (durées d'ingestion et de rumination) des vaches laitières (tableau 4). En revanche, dans un essai de Hindhede et al (1996) sur des génisses laitières, l'augmentation de la densité de 3 à $1,5 \mathrm{~m}^{2}$ par animal, sans qu'il y ait de compétition alimentaire, s'est traduite par une diminution de la vitesse de croissance de $174 \mathrm{~g} / \mathrm{j}$ (soit $31 \%$ ) pour des quantités ingérées identiques. Dans une expérience similaire avec 120 taurillons laitiers, Andersen et al (1997) ont également observé des effets importants des niveaux de densité sur la vitesse de crois-

\section{$L$ a répartition quotidienne des activités d'ingestion dans un troupeau dépend du nombre d'accès possibles aux aliments, sans que les quantités ingérées ne soient modifiées.}


Tableau 4. Durées d'ingestion, de rumination et de couchage pour des vaches laitières selon les périodes de la journée et effet de la place disponible dans la stabulation libre : 13,9 (G) ou 2,5 (P) $\mathrm{m}^{2}$ par animal (d'après Metz et Mekking 1984).

\begin{tabular}{|c|c|c|c|}
\hline & Duré & ivité & Ecart $\mathbf{G}-\mathbf{P}$ \\
\hline & $\min$ & $\%$ & $\min$ \\
\hline $\begin{array}{l}\text { Ingestion } \\
09 \mathrm{~h} 30 \text { à } 15 \mathrm{~h} 30 \\
18 \mathrm{~h} 00 \text { à } 20 \mathrm{~h} 00 \\
22 \mathrm{~h} 00 \text { à } 02 \mathrm{~h} 30\end{array}$ & $\begin{array}{l}93,5 \\
39,4 \\
21,2\end{array}$ & $\begin{array}{c}26,0 \\
32,8 \\
7,9\end{array}$ & $\begin{array}{l}-2,5 \\
-1,5 \\
-5,4\end{array}$ \\
\hline $\begin{array}{l}\text { Rumination } \\
09 \mathrm{~h} 30 \text { à } 15 \mathrm{~h} 30 \\
18 \mathrm{~h} 00 \text { à } 20 \mathrm{~h} 00 \\
22 \mathrm{~h} 00 \text { à } 02 \mathrm{~h} 30\end{array}$ & $\begin{array}{c}142,3 \\
38,8 \\
137,6\end{array}$ & $\begin{array}{l}39,6 \\
32,3 \\
51,0\end{array}$ & $\begin{array}{l}-4,8 \\
-3,6 \\
-3,3\end{array}$ \\
\hline $\begin{array}{l}\text { Position couchée } \\
09 \mathrm{~h} 30 \text { à } 15 \mathrm{~h} 30 \\
18 \mathrm{~h} 00 \text { à } 20 \mathrm{~h} 00 \\
22 \mathrm{~h} 00 \text { à } 02 \mathrm{~h} 30\end{array}$ & $\begin{array}{c}191,9 \\
42,6 \\
199,5\end{array}$ & $\begin{array}{l}53,3 \\
35,5 \\
73,9\end{array}$ & $\begin{array}{l}-4,4 \\
-7,8 \\
-2,2\end{array}$ \\
\hline
\end{tabular}

sance et l'indice de consommation : 5 à $12 \%$ en faveur de la densité la plus faible. Wierenga et al (1985) ont analysé les conséquences d'une augmentation de l'espace offert sur le comportement de vaches laitières conduites en stabulation à logettes au-delà d'une situation jugée "normale ». Les durées d'ingestion et le temps passé en position couchée ont été supérieurs chez les vaches disposant du plus grand espace. L'augmentation de la durée d'ingestion a eu lieu pendant les phases principales d'ingestion quotidienne (grands repas). Globalement, les vaches ont été plus synchronisées quand elles avaient davantage d'espace disponible.

Dans une revue résumant un grand nombre d'essais, Ingvartsen et Andersen (1993) concluent que la réduction de l'espace disponible pour des bœufs et des taurillons de 250 à $500 \mathrm{~kg}$ de poids vif diminue leur niveau d'ingestion et leur vitesse de croissance et augmente leur indice de consommation : respectivement 92 - 81 et $115 \%$ avec 1,5 vs $4,7 \mathrm{~m}^{2}$ par animal. De la même façon, chez des taurillons disposant de 5,67 $\mathrm{m}^{2}$ au lieu de $1,81 \mathrm{~m}^{2}$ par animal, la vitesse de croissance a été augmentée de $25 \%$ (Ilan et al 1979)., Les résultats sont comparables à ceux des génisses : les vitesses de croissance sont moindres pour les densités les plus fortes : $520-650-700$ et $690 \mathrm{~g} / \mathrm{j}$, respectivement avec $1,5-2-2,5$ et $3 \mathrm{~m}^{2}$ disponibles par animal (génisses croisées Simmental en finition ; Fisher et al 1997).

Les niveaux de densité recommandés pour des bovins conduits en bâtiment dépendent du type de sol ainsi que du poids ou de l'âge des animaux (Lawrence 1994). Les valeurs en vigueur dans les pays d'Europe de l'Ouest pour les bovins en croissance sont en moyenne de 1 et $0,75 \mathrm{~m}^{2}$ pour $100 \mathrm{~kg}$ de poids vif, respectivement en stabulation avec aire paillée ou sur caillebotis uniquement, même si ces valeurs sont parfois très variables (Mossberg 1994). II ne semble pas que cela induise de différence significative d'activité d'ingestion et de repos comme l'a montré Hardy (1980) chez des bœufs à l'engrais, ni des performances de croissance chez des taurillons (Mossberg et al 1993).
En conclusion, les contraintes importantes que les bovins peuvent subir au sein du groupe sont une réduction de l'espace disponible et du temps d'accès à la nourriture. Ces contraintes engendrent une diminution de la durée d'ingestion et de la synchronisation de l'activité alimentaire, associée à une augmentation de la vitesse d'ingestion pouvant permettre de maintenir les quantités ingérées même lorsque le niveau de compétition est élevé. L'amplitude de ces effets dépend de facteurs liés aux aliments (ingestibilité) et aux animaux (race, âge, poids, sexe, niveau de production). L'espace disponible pour le groupe, indépendamment de l'alimentation, est un facteur à ne pas négliger. En deçà d'un certain niveau (moins de $3 \mathrm{~m}^{2}$ par animal pour des jeunes bovins en croissance), le niveau d'ingestion et donc la vitesse de croissance (figure 6) peuvent être diminués, notamment pour les animaux dominés.

Figure 6. Vitesse de croissance de bœufs, taurillons et génisses conduits en stabulation libre en fonction de la densité (d'après Hardy 1980, Ingvartsen et Andersen 1993, Hindhede et al 1996, Andersen et al 1997, Fisher et al 1997).

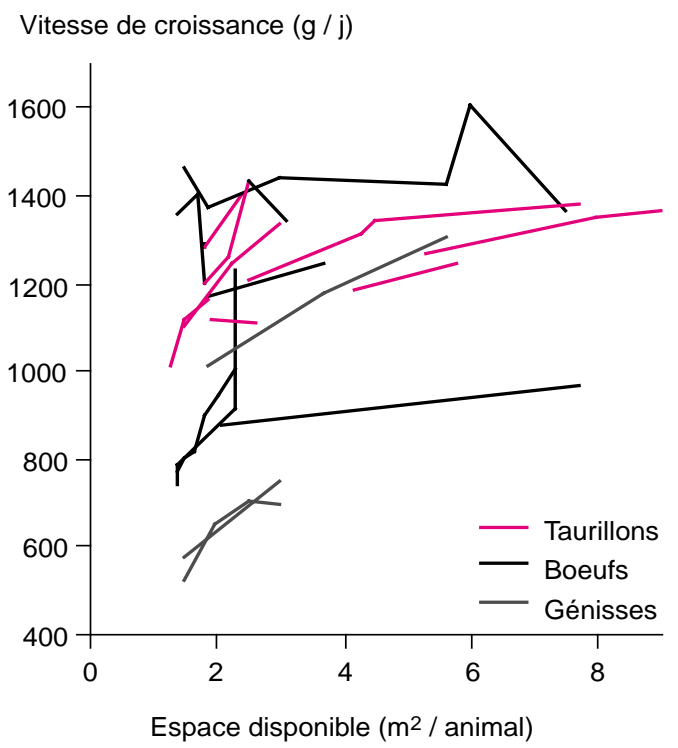




\section{4 / Taille et stabilité des groupes}

\section{1 / Taille des groupes}

Des essais spécifiques ont été menés avec des ovins pour déterminer la taille minimale des groupes à former lors de la mise en place de protocoles expérimentaux afin d'obtenir des résultats fiables et représentatifs en raison de l'aspect fortement grégaire de l'espèce (voir par exemple Penning et al 1993). Ce type d'approche n'a pas été réalisé pour les bovins. Par ailleurs, peu d'essais spécifiques ont visé à définir la taille optimale des groupes de bovins dans les élevages (Lawrence 1994). Pour cet auteur, la taille optimale ne devrait pas dépasser 20 têtes pour des animaux disposant d'une aire paillée et devrait être comprise entre 10 et 20 têtes dans les autres cas (sols en pente). Les raisons invoquées sont de deux ordres : la surveillance des individus par l'éleveur est plus facile et la structure sociale est plus stable et plus rapidement établie.

Hindhede et al (1996) n'ont pas détecté de différences de vitesse de croissance, ni de quantité ingérée pour des génisses laitières conduites en groupes de 6 ou 12 têtes. Avec des vaches laitières à haut potentiel et sur des périodes expérimentales courtes ( 14 jours), la taille des groupes n'a pas d'effet, tant sur les niveaux de production laitière que sur les rythmes circadiens d'activité alimentaire et de repos (Szucs et al 1991). Seule la durée quotidienne d'ingestion a diminué avec l'augmentation des effectifs par groupe : $5 \mathrm{~h} 26$ vs 4 h59, respectivement pour 30 et 80 têtes en stabulation libre avec aire d'exercice (12,2 $\mathrm{m}^{2} /$ animal) et $6 \mathrm{~h} 39$ vs $5 \mathrm{~h} 37$, respectivement pour 15 et 70 têtes en stabulation libre sans aire d'exercice $\left(4,4 \mathrm{~m}^{2} / a n i m a l\right)$. Sur la base de quatre essais portant sur l'effet de la densité, de la taille du groupe et de la taille de la case de stabulation pour des bœufs croisés à l'engrais, Morrison et al (1981) n'ont pas mis en évidence d'effet systématique de la taille des groupes (de 5 à 20 têtes) ou de la densité ( 1,5 à $7,5 \mathrm{~m}^{2}$ par animal) sur le niveau d'ingestion, la vitesse de croissance et l'indice de consommation.

\section{2 / Stabilité des groupes}

Lorsque le troupeau est structuré en lots, les valeurs individuelles associées au critère de répartition des animaux dans ces lots peuvent évoluer dans le temps. C'est par exemple le cas pour la production laitière individuelle, l'état corporel ou encore l'intervalle entre la mise bas et la saillie fécondante. Le maintien de l'homogénéité intra-lot pour ce critère nécessite alors de modifier régulièrement la composition des lots par transfert d'animaux (Clark et al 1977, Albright 1991). La question est alors de savoir quelle incidence vont avoir ces transferts sur le comportement et les performances des animaux et quel est le meilleur compromis entre la fréquence des transferts et I'homogénéité intra-lot pour un critère donné.

Des génisses ingèrent davantage quand elles sont en compétition avec des animaux issus de leur propre lot d'élevage qu'avec des animaux inconnus, les dérangements subis et les réactions agressives étant moins nombreux dans le premier cas (Bouissou et Hövels 1976), et ce jusqu'à un an après le regroupement des différents lots. Des génisses laitières introduites dans le troupeau reproducteur par groupes de 3 animaux ont moins d'interactions avec les multipares que celles introduites individuellement (Knierim 1998), mais la différence disparaît au bout de 4 jours. Un essai réalisé avec des groupes de 19 vaches de race à viande en lactation au sein desquels a été introduite une vache provenant d'un autre élevage, a montré un effet
Les performances sont peu affectées par la taille du groupe, en revanche elles peuvent être diminuées lorsque les animaux changent de groupe.

Figure 7. Production laitière de vaches primipares selon qu'elles changent de groupe ou qu'elles restent dans le groupe ; moyennes mobiles sur 3 jours (adapté d'Hasegawa et al 1997).

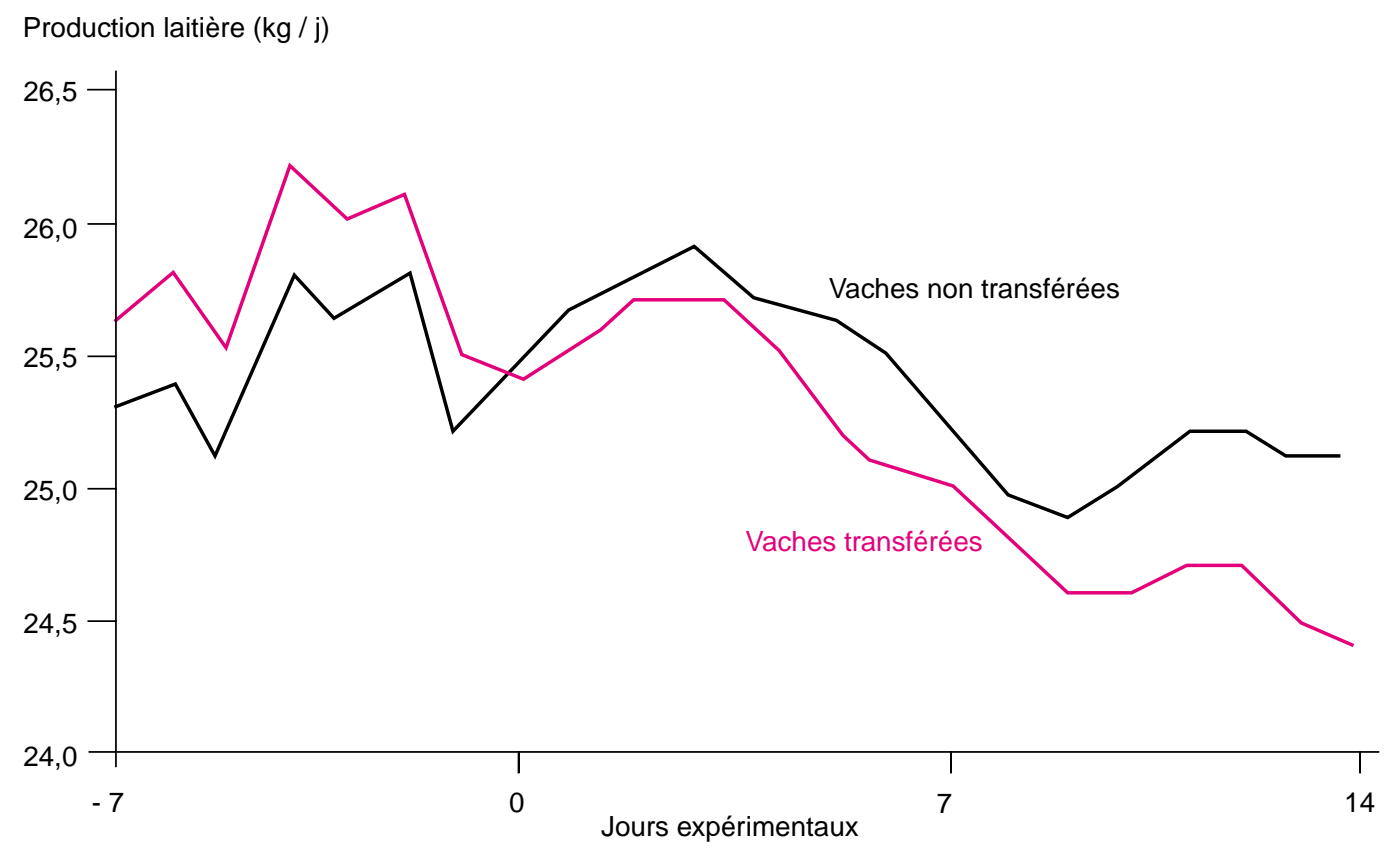


cumulatif du rang social et de l'origine extérieure de l'animal sur le stress social (Mench et al 1990) : I'animal étranger a été d'autant plus désavantagé qu'il était de rang social faible, mais cela n'a pas eu d'effet sur ses paramètres de production (poids vif et gain de poids des veaux). Les auteurs pensent que cela n'aurait pas été le cas avec des groupes d'effectifs plus importants. D'ailleurs, Hasegawa et al (1997) ont observé une diminution de la production laitière sur des vaches primipares après les avoir changées de groupe (figure 7), et ce d'autant plus qu'elles étaient de faible rang social.

\section{Conclusion}

Le niveau d'ingestion est une composante de la performance animale dans la mesure où il conditionne la quantité de nutriments disponibles pour la production. La modification des paramètres du comportement alimentaire rend compte des capacités d'adaptation des individus face aux contraintes qu'ils subissent au sein du groupe (rang social des congénères, espace disponible, compétition alimentaire) ou au contraire aux stimuli engendrés par d'autres individus du groupe (effet d'entraînement).

Quand ils sont conduits à l'attache, et donc alimentés individuellement, les bovins ingèrent souvent moins que quand ils sont conduits en groupe. Ces résultats constituent un premier indicateur d'un " effet groupe » sur le comportement alimentaire. Ils ont été obtenus avec des rations très ingestibles distribuées à des animaux à besoins élevés (bovins en croissance et vaches laitières), induisant une forte motivation à ingérer. Les performances (vitesse de croissance ou production laitière) ne sont pas affectées dès lors que la compétition alimentaire est inexistante ou faible.
La hiérarchie sociale s'établit rapidement et reste stable dans le temps, même lorsque de nouveaux individus sont introduits dans le groupe. La synchronisation des activités est un phénomène très robuste tant au pâturage qu'en bâtiments, mais qui peut être affecté dans des conditions défavorables (compétition alimentaire, forte densité). La taille et la stabilité du groupe (fréquence des transferts d'animaux d'un groupe à l'autre), semblent avoir peu voire pas d'effet sur le niveau d'ingestion et les paramètres du comportement alimentaire. Cependant, la majorité des essais concernent des vaches laitières (en particulier de race Holstein), sans doute moins réactives que des vaches de race à viande (Le Neindre et Sourd 1984).

Le temps d'accès à l'auge est le facteur le plus déterminant pour le maintien du niveau d'ingestion. Quand il est limité pour tout le groupe à l'échelle de la journée, les animaux augmentent leur vitesse d'ingestion. Quand il est limité en raison de la compétition alimentaire (largeur et/ou nombre d'auges), en liaison avec la taille du groupe, les animaux peuvent s'adapter en modifiant leur rythme d'activité : fractionnement et décalage des repas dans le temps, avec comme conséquence la désynchronisation de l'activité alimentaire au sein du groupe (figure 8). L'espace disponible à l'auge est un critère beaucoup plus influent sur les performances et le comportement alimentaire que l'espace total disponible dans la case de stabulation libre. Ainsi, l'effet défavorable de la densité sur la croissance de jeunes bovins n'apparaît qu'en deçà de 2 à $3 \mathrm{~m}^{2}$ par animal.

La variabilité individuelle des formes d'adaptation comportementale au sein du groupe n'a pas été systématiquement étudiée excepté pour le statut hiérarchique qui consti-

Figure 8. Relations entre les paramètres du comportement alimentaire des bovins et les facteurs de l'environnement liés à la conduite en groupe. Les signes + indiquent une corrélation positive, les signes - une corrélation négative.

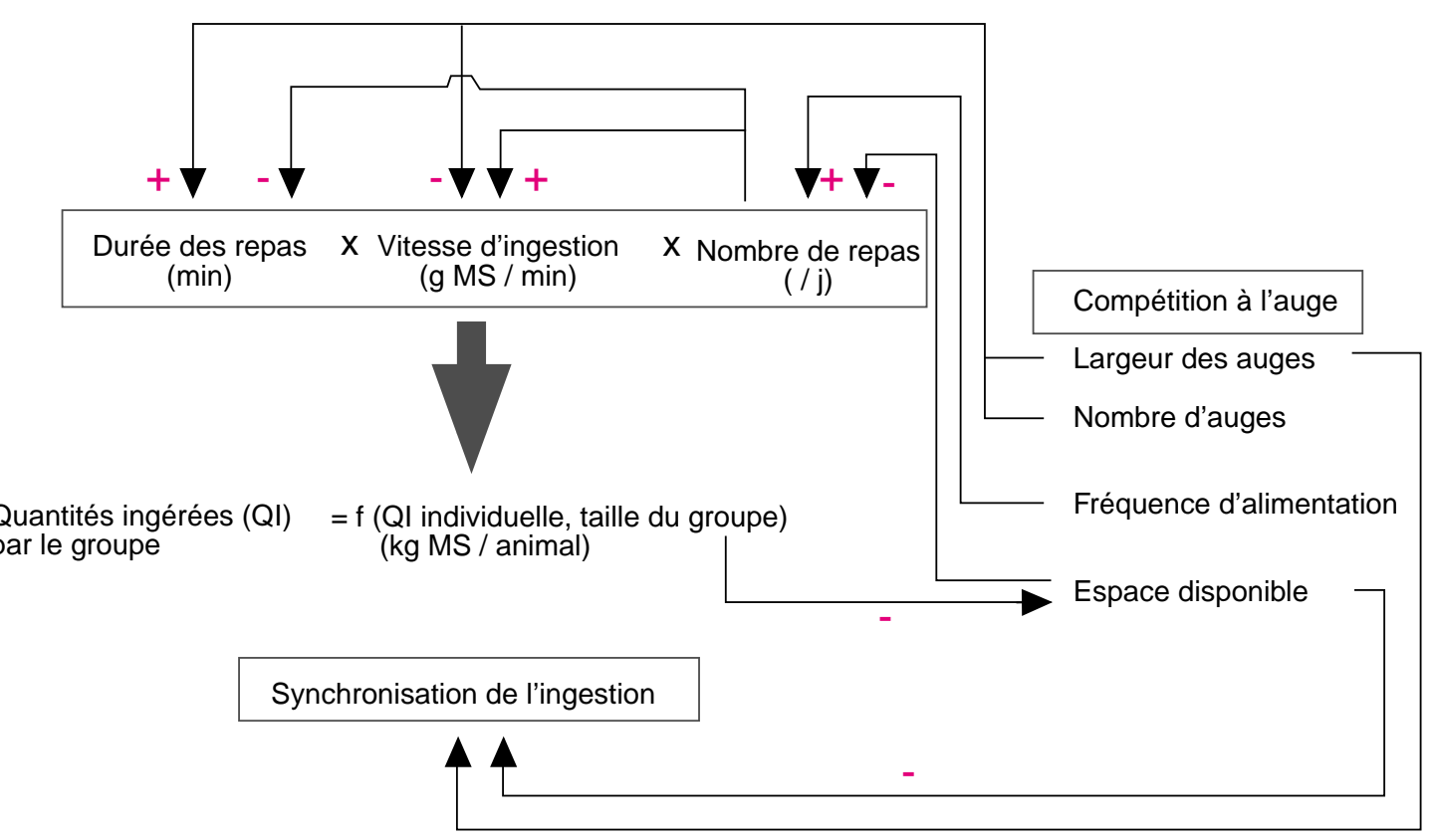


tue parfois une clé d'interprétation des résultats ; par exemple, les individus dominants ont une durée d'ingestion quotidienne supérieure à celle des dominés, lesquels tendent à accroître leur vitesse d'ingestion. Cette clé d'interprétation reste valable quelle que soit la stratégie d'affectation des animaux dans les lots : en effet, dans chaque lot constitué, il y aura toujours des individus dominants ou dominés par rapport aux autres.

Le niveau des besoins nutritionnels des animaux composant le groupe conditionne également leur capacité d'adaptation (J ournet et
Rémond 1976, Friend et al 1977, Metz et Wierenga 1987), mais les effets de I'hétérogénéité de ces besoins sur les paramètres du comportement alimentaire n'ont pas été étudiés. Les groupes expérimentaux sont d'ailleurs généralement constitués pour être les plus homogènes possibles. Nos résultats (Ingrand 1999) ainsi que ceux cités précédemment montrent pourtant que le niveau de besoins nutritionnels est un facteur majeur d'orientation des adaptations du comportement alimentaire, probablement prioritaire par rapport au rang social.

\section{Références}

Albright J L., 1971. Effects of varying the environment on the behaviour and performance of dairy cattle. Proc. N.Z. Soc. Anim. Prod., 31, 10-21.

Albright J.L., 1991. To group or not to group. Beef/Dairy Day Report. Purdue University, West Lafayette, Indiana. 45-50.

Albright J .L., 1993. Nutrition, feeding, and calves. Feeding behavior of dairy cattle. J. Dairy Sci., 76, 485-498.

Albright J.L., Arave C.W., 1997. The behaviour of cattle. CAB International, $306 \mathrm{p}$.

Andersen H.R., J ensen L.R., Munksgaard L., Ingvartsen K.L., 1997. Influence of floor space allowance and access sites to feed trough on the production of calves and young bulls and on the carcass and meat quality of young bulls. Acta Agric. Scand. Sect. A, Anim. Sci., 47, 48-56.

Arave C.W., Mickelsen C.H., Lamb R.C., Svejda A.J., Canfield R.V., 1974. Effect of social status, age, and body weight on plasma total corticoids of dairy cattle. J. Dairy Sci., 57, 5, 629-630.

Arave C.W., Albright J .L., Armstrong D.V., Foster W.W., Larson L.L. 1992. Effects of isolation of calves on growth, behavior, and first lactation milk yield of Holstein cows. J. Dairy Sci., 75, 3408-3415.

Beilharz R.G., Butcher D.F., Freeman, A.E., 1966. Social dominance and milk production in Holsteins. J. Dairy Sci., 49, 887-892.

Bines J.A., Davey W.F., 1970. Voluntary intake, digestion, rate of passage, amount of material in the alimentary tract and behaviour in cows receiving complete diets containing straw and concentrates in different proportions. Br. J. Nutr., 24, 1013-1027.

Blanc F., Thériez M., Brelurut A., 1999. Effects of mixedspecies stocking and space allowance on the behaviour and growth of red deer hinds and ewes at pasture. Appl. Anim. Behav. Sci., 63, 41-53.

Bocquier F., Guillouet P., Barillet F., 1995. Alimentation hivernale des brebis laitières : intérêt de la mise en lots. INRA Prod. Anim., 8, 1-10.

Bouissou M.F., 1965. Observations sur la hiérarchie sociale chez les bovins domestiques. Ann. Biol. Anim. Bioch. Biophys., 5, 327-339.

Bouissou M.F., Hövels J.H., 1976. Effet des conditions d'élevage sur le comportement des génisses dans une situation de compétition alimentaire. Ann. Zootech., 25, 2, 213-219.
Broadbent P.J ., Mclntosh J .A.R., Spence A., 1970. The evaluation of a device for feeding group-housed animals individually. Anim. Prod., 12, 245-252.

Campling R.C., Morgan C.A., 1981. Eating behaviour of housed dairy cows: a review. Dairy Sci. Abstracts, 43, 2, 57-63.

Clark P.W., Ricketts R.E., Krause G.F., 1977. Effect on milk yield of moving cows from group to group. J. Dairy Sci., 60, 769-772.

Coppock C.E., Noller C.H., Crowl B.W., McLellon C.D., Rhykerd C.L., 1972. Effect of group versus individual feeding of complete rations on feed intake of lactating cows. J. Dairy Sci., 55, 325-327.

Corkum M.J., Bate L.A., Tennessen T., Lirette A., 1994. Consequences of reduction of number of individual feeders on feeding behaviour and stress level of feedlot steers. Appl. Anim. Behav. Sci., 41, 27-35.

Dalton D.C., Pearson M.E., Sheard M., 1967. The behaviour of dairy bulls kept in groups. Anim. Prod., 9, 1-5.

Dantzer R., Mormède P., 1979. Le stress en élevage intensif. Actualités scientifiques et agronomiques de I'INRA. Masson, Paris, 117 p.

D'Hour P., Petit M., Garel J.P., 1991. Components of grazing behaviour of 3 breeds of heifers. Ann. Zootech. 44, (Suppl. 1), 270.

Durst B., Senn M., Langhans W., 1993. Eating pattern of lactating dairy cows of three breeds fed grass ad lib. Phys. Behav., 54, 625-631.

Fisher A.D., Crowe M.A., O'Kiely P.O., Enright W.J ., 1997. Growth, behaviour, adrenal and immune responses of finishing beef on slatted floors at $1.5,2.0,2.5$ or $3.0 \mathrm{~m}$ ? space allowance. Livest. Prod. Sci., 51, 245-254.

Friend T.H., Polan C.E., 1974. Social rank, feeding behavior, and free stall utilization by dairy cattle. J. Dairy Sci., $57,1214-1220$

Friend T.H., Polan C.E., McGilliard M.L., 1977. Free stall and feed bunk requirements relative to behavior, production and individual feed intake in dairy cows. J. Dairy Sci., $60,108-116$.

Gonyou H.W., Stricklin W.R., 1981. Eating behaviour of beef cattle groups fed from a single stall or trough. Appl. Anim. Ethol., 7, 123-133.

Gonyou H.W., Stricklin W.R., 1984. Diurnal behavior patterns of feedlot bulls during winter and spring in northern latitudes. J. Anim. Sci., 58, 1075-1083. 
Gustafson G.M., Luthman I. Burstedt E., 1993. Effect of daily exercise on performance, feed efficiency and energy balance of tied dairy cows. Acta Agric. Scand. Sect. A, Anim. Sci., 43, 219-227.

Hanekamp W.J .A., Smits A.C., Wierenga H.K., 1994. Open versus closed barn and individual versus group-housing for bull calves destined for beef production. Livest. Prod. Sci., 37, 261-270.

Harb M.Y., Reynolds V.S., Campling R.C., 1985. Eating behaviour, social dominance and voluntary intake of silage in group-fed milking cattle. Grass F orage Sci., 40, 113-118.

Hardy R., 1980. Observations on the performance and behaviour of finishing steers kept on two housing systems. Anim. Prod., 30, 456.

Hasegawa N., Nishiwaki A., Sugawara K., Ito I., 1997. The effects of social exchange between two groups of lactating primiparous heifers on milk production, dominance order, behavior and adrenocortical response. Appl. Anim. Behav. Sci., 51, 15-27.

Hindhede J Sorensen J T. J ensen M. B. Krohn C. C. 1996. Effect of space allowance, access to bedding, and flock size in slatted floor systems on the production and health of dairy heifers. Acta Agric. Scand. Sect A, Anim. Sci., 46, 46-53.

Ilan D., Bleiberg M., Holzer Z., Levy D., Folman Y., 1979. The effect of floor space allowance on feedlot performance and on behaviour of Israeli-Friesian male cattle. Livest. Prod. Sci., 6, 307-312.

Ingrand S., 1999. Constitution des lots de vaches dans les élevages allaitants. Effets de l'hétérogénéité des besoins nutritionnels sur les quantités ingérées et le comportement alimentaire. Thèse Doct-Ing. de I'INA-ParisGrignon, 262 p.

Ingrand S., Agabriel J ., Lassalas J ., Dedieu B. 1999. How group feeding influences intake level of hay and feeding behaviour of beef cows. Ann. Zootech., 48, 435-445.

Ingvartsen K.L., Andersen H.R., 1993. Space allowance and type of housing for growing cattle. A review of performance and possible relation to neuroendocrine function. Acta Agric. Scand. Sect. A, Anim. Sci., 43, 65-80.

J ournet M., Rémond B., 1976. Physiological factors affecting the voluntary intake of feed by cows: a review. Livest. Prod. Sci., 3, 129-146.

Knierim U., 1998. Social and resting behaviour of heifers after single or group introduction in the dairy herd. In : I. Veissier et A. Boissy (eds), Proc. 32nd Congress of the International Society for Applied Ethology, 153. INRA, Paris.

Kondo S., 1987. Behavioral adaptation in group of cattle. Memoirs of the Faculty of Agriculture Hokkaido University Sapporo, J apan, 15, 192-233.

Krohn C.C., 1994. Behaviour of dairy cows kept in extensive (loose housing/pasture) or intensive (tie stall) environments. III. Grooming exploration and abnormal behaviour. Appl. Anim. Behav. Sci., 42, 73-86.

Krohn C.C., Munksgaard L., J onasen B., 1992. Behaviour of dairy cows kept in extensive (loose housing/pasture) or intensive (tie stall) environments. I. Experimental procedure, facilities, time budgets - diurnal and seasonal conditions. Appl. Anim. Behav. Sci., 34, 37-47.

Lawrence N.G., 1994. Beef cattle housing. In : C.M. Wathes and D.R. Charles (eds), Livestock housing, 339357. CAB International.

INRA Producti ons Ani males, jui llet 2000
Leaver J.D., Yarrow N.H., 1980. A note on the effect of social rank on the feeding behaviour of young cattle on self-feed maize silage. Anim. Prod., 30, 303-306.

Le Neindre P., Sourd C., 1984. Influence of rearing conditions on subsequent social behaviour of Friesian and Salers heifers from birth to six months of age. Appl. Anim. Behav. Sci., 12, 43-52.

Manson F.J. Appleby M.C. 1990. Spacing of dairy cows at a food trough. Appl. Anim. Behav. Sci., 26, 69-81.

Mench J.A., Swanson J.C., Stricklin W.R., 1990. Social stress and dominance among groups members after mixing beef cows. Can. J. Anim. Sci., 70, 345-354.

Mestertongibbons M., Dugatkin L.A., 1995. Toward a theory of dominance hierarchies: effects of assessment, group size, and variation in fighting ability. Behav. Ecol., 6, 416-423.

Metz J.H.M., Mekking P., 1984. Crowding phenomena in dairy cows as related to available idling space in a cubicle housing system. Appl. Anim. Behav. Sci., 12, 63-78.

Metz J.H.M., Wierenga H.K., 1987. Behavioural criteria for the design of housing systems for cattle. In . H.K. Wierenga et D.J. Peterse (eds), Cattle housing systems, lameness and behaviour, 14-25. Martinus Nijhoff Publishers.

Miller K., Wood-Gush D.G.M., 1991. Some effects of housing on the social behaviour of dairy cows. Anim. Prod. 53, 271-278.

Morrison S.R., Lofgreen G.P., Prokop M., 1981. Effect of floor space allotment and animal group size on beef cattle performance. Transactions of the American Society of American Engineers, 24, 450-451.

Mossberg I., 1994. Housing systems for growing bulls. Do they get what they need ?. In Proceedings of the 45th Annual Meeting of the European Association for Animal Production, Edinburgh, United Kingdom, September 1994.

Mossberg L., Lindell L., ohnsson S., Törnquist M., 1993. Insulated and uninsulated housing systems for growing bulls fed grass silage ad libitum. Acta Agric. Scand. Sect. A, Anim. Sci., 43, 107-115.

Munksgaard L., Simonsen H.B., 1995. Behavioural and pituitary-adrenal axis responses of tethered cows or cows kept in pens with slatted floors. Acta Agric. Scand. Sect. A, Anim. Sci., 45, 132-138.

Nour A.Y.M.. Thonney M.L., 1987. Carcass, soft tissue and bone composition of early and late maturing steers fed two diets in two housing types and serially slaughtered over a wide weight range. J. Agric. Sci., 109, 345-355.

Penning P.D., Parsons A.J ., New man J .A., Orr R.J., Harvey A., 1993. The effects of group size on grazing time in sheep. Appl. Anim. Behav. Sci., 37, 101-109.

Phipps R.H., Bines J.A., Cooper A., 1983. A preliminary study to compare individual feeding through calan electronic feeding gates to group feeding. Anim. Prod., 36, 544.

Rook A.J., Huckle C.A., 1995. Synchronization of ingestive behaviour by grazing dairy cows. Anim. Sci., 60, 25-30

Stricklin W.R., Gonyou H.W., 1981. Dominance and eating behaviour of beef cattle fed from a single stall. Appl. Anim. Ethol., 7, 135-140.

Syme G.J., Syme L.A., 1975. The concept of spatial leadership in farm animals: an experiment with sheep. Anim. Behav., 23, 921-925. 
Szucs E., Acs I., Ugry K., Csiba A., 1991. Effect of group size on the performance and comfort behaviour of dairy cows of high genetic potential. In Proceedings of the 42th Annual Meeting of the European Association for Animal Production, Berlin, 9th-12th September 1991, Volume 1, 509.

Welch J.G., Hooper A.D., 1988. Ingestion of feed and water. In : D.C. Church (ed), The Ruminant Animal. Digestive physiology and nutrition, 108-116. Prentice Hall, New Jersey 07632
Wierenga H.K., 1990. Social dominance in dairy cattle and the influences of housing and management. Appl. Anim. Behav. Sci., 27, 201-229.

Wierenga H.K., Metz J.H.M., Hopster H., 1985. The effect of extra-space on the behaviour of dairy cows kept in a cubicle house. In : R. Zayan (ed), Social space for domestic animals, 160-169. A seminar in the CEC programme of coordination of research on animal welfare, Brussels, January 10-11. Martinus Nijhoff Publishers, Dordrecht/Boston/Lancaster.

\section{Abstract}

Feeding behaviour, intake and performance in beef cattle managed in groups.

Social hierarchy and leadership are two major components of social behaviour, which strongly influence intake and feeding behaviour of cattle managed in groups. In animals with high nutritional requirements (dairy or growing cattle) this effect is characterised by higher intake and lower feed conversion compared to situations where animals are individually fed (tie-stall systems or individual pens). The characteristics and environment of the animal groups (competition for food, density, number of feeders, animal number and characteristics, stability in group composition) also induce certain effects that we have attempted to quantify. Increasing density and decrease of width at trough or time of access to food induce a decrease in daily duration and synchronisation of eating, with an atten- dant increase in the eating rate which may help maintain the intake level, even with a high level of competition for food. Group size has little effect on intake and feeding behaviour; it only plays a role in dictating the time required for the social hierarchy to be established. Animals with high nutritional requirements have higher intake and eating rates and longer daily duration of eating than those with lower requirements, but the effect of within-group heterogeneity of nutritional requirements has not been assessed. Our on-farm studies have shown, however, that in most cases the withingroup nutritional requirements of the beef cattle groups managed by the farmers are not homogenous. This raises the question of the potential deterioration of performances in animals kept in such heterogeneous groups.

INGRAND S., 2000. Comportement alimentaire, quantités ingérées et performances des bovins conduits en groupe. INRA Prod. Anim., 13, 151-163. 